\title{
Análisis cualitativo-cuantitativo, gestión de la Coordinación Académica del Curso Nivelación 2017 (2) perspectiva del estudiante
}

\section{Qualitative-quantitative analysis, management of the Academic Coordination, Leveling Course 2017 (2) student perspective}

Análisis la Gestión de la Coordinación Académica, 2017 (2)

Joe González $\operatorname{Arcos}^{(1)}$

Jackeline Pazmiño Castro ${ }^{(2)}$

Johnny Rodríguez ${ }^{(3)}$

Luz Marina Banchón ${ }^{(4)}$

(1) Profesional independiente. Ecuador. e-mail: joegonzalez_7@hotmail.com

(2) Profesional independientes. Ecuador. e-mail: jackyalexandrap.7@ outlook.es

(3) Universidad Laica Eloy Alfaro de Manabí. Ecuador. e-mail: johnrod.docente@ gmail.com

(4) Unidad Educativa Pedro Balda Cucalón. Ecuador. e-mail: many_1011@ @hotmail.es

Contacto:joegonzalez_7@hotmail.com

\section{Recibido: 20-7-2020}

Aprobado: 30-11-2020

\section{Resumen}

El presente artículo tiene por objetivo, evaluar la gestión de la coordinación académica y determinar el grado de satisfacción de los estudiantes en el proceso de nivelación en los cursos de nivelación de la Universidad Laica Eloy Alfaro de Manabí, Ecuador. Se trabajo bajo un enfoque cualitativo-cuantitativodescriptivo-taxonómico, con análisis de cada una de las variables. Los principales resultados evidencian aspectos determinantes con la calidad, referida esta a las frágiles condiciones de infraestructura, acceso a los medios didácticos y tecnológicos mínimos necesarios. Se evidencia que el $91 \%$ de los estudiantes estuvieron conformes en lo relativo a los temas aprendidos y su utilidad para los educandos en el 
aprendizaje. Se constata el empleo de contrapesos como estrategia de gestión que atenúan el impacto de las deficiencias y finalmente, se confirman que las respuestas en la evaluación a los estudiantes, resultan contradictorias ante una realidad observable, propiciando su análisis en posteriores investigaciones.

Palabras clave: Coordinación Académica, evaluación de la gestión, contrapesos.

\section{Summary}

The objective of this article is to evaluate the management of academic coordination and determine the degree of satisfaction of students in the leveling process in leveling courses at the Universidad Laica Eloy Alfaro de Manabí, Ecuador. Work was done under a qualitative-quantitative-descriptive-taxonomic approach, with analysis of each of the variables. The main results show determining aspects with quality, referring to the fragile conditions of infrastructure, access to the minimum necessary didactic and technological means. It is evidenced that $91 \%$ of the students were satisfied with regard to the topics learned and their usefulness for the learners in learning. The use of counterweights as a management strategy that mitigates the impact of the deficiencies is verified and finally, it is confirmed that the answers in the evaluation to the students are contradictory before an observable reality, favoring their analysis in subsequent investigations.

Key words:Academic Coordination, management evaluation, counterweights.

\section{Introducción}

La evaluación es parte de una globalización galopante que obliga a las organizaciones a mirar hacia dentro y emprender transformaciones permanentes. Los desafíos de las organizaciones tienen implicación directa con la calidad de sus servicios, e implícitamente con la calidad de sus procesos y sub procesos, dar respuestas pertinentes y oportunas es la tarea principal, ante las nuevas exigencias sociales de formación profesional.

Según Moreno (2016), relata que no existen respuestas buenas ni malas, sino distintos niveles de aproximación a unos criterios de evaluación que definen lo que implica haber logrado una comprensión a fondo de la información. El autor afirma que se requiete comprender la importancia del establecimiento de criterios que conducirán hacia el objetivo mismo de la evaluación. entonces, se los debe sumir como 
la herramienta a través de la cual se procederá a acceder a una realidad concreta. Así mismo, García (2010) se expresa de la siguiente manera respecto de los criterios de evaluación, deben concretarse en distintas dimensiones, subdimensiones y atributos que permitan medir de manera más precisa la evolución en el aprendizaje del alumno, su nivel y calidad,

Por consiguiente, el ¿qué, para qué, cómo y a quienes evaluar? orientan el camino que transitará en todo el proceso, tiene que ver con la determinación del objetivo; obtener una panorámica del cumplimiento de los objetivos educacionales; la metodología que se aplicará y finalmente determinar el grupo al que se aplicarán los instrumentos elegidos para este efecto. De no existir estos criterios, cómo se podrían determinar las acciones para la mejora si no se conocen los problemas existentes y no se los aborda con claridad.

Como se puede advertir, la evaluación es parte sustantiva y transversal de toda gestión educativa. Implica una interpretación concreta de la realidad y una toma de conciencia de los resultados, su objetividad dependerá en gran medida justamente de los criterios que la sustenten. No contar con criterios, involucraría un desconocimiento del vehículo idóneo que conduciría al objetivo de la evaluación. En este sentido Palladino y Palladino (1998), indica que la gestión incluye la acción y el efecto de administrar de manera tal que se realicen diligencias conducentes al logro apropiado de las respectivas finalidades de las instituciones.

El presente artículo tiene por objetivo, evaluar la gestión de la coordinación académica y determinar el grado de satisfacción de los estudiantes del proceso de nivelación 2017 (2) cuyos resultados, de emplearse de forma adecuada, podrían mejorar las condiciones de enseñanza aprendizaje en los futuros cursos de nivelación de la Universidad Laica Eloy Alfaro de Manabí.

\section{Materiales y métodos}

La investigación se realizó en la Universidad Laica Eloy Alfaro de Manabí, a través de la Dirección de Admisión y Nivelación realiza el levantamiento de la información de la calidad de la gestión de su Coordinación Académica en el período 2017 (2). Para realizar la recolección de la información se empleó la técnica de la encuesta,la misma que fue diseñada con los criterios de evaluación elaborados por la comisión conformada para este efecto, dicho instrumento consta de tres apartados, el primero 
“Organización” que mide aspectos tales como: Organización del curso de nivelación, condiciones del aula para el aprendizaje, cantidad de estudiantes por curso, horario del curso y atención al estudiante.

Con el segundo apartado, "Actividad Formativa" con la cual se pretende evaluar el proceso de enseñanza-aprendizaje,en el que se incluyen los siguientes aspectos: los contenidos han cubierto sus expectativas; los temas, se han tratado con la profundidad esperada; importancias de las asignaturas recibidas, adquisición de conocimientos y la percepción sobre la utilidad de los temas aprendidos. Finalmente, el tercer apartado evalúa de forma global al curso de Nivelación 2017 II.

El instrumento consta de diez ítems, se emplea la escala de Likert con cuatro opciones de respuesta, siendo esta de tipo psicométrica, puesto que admite el sentir del estudiante, basado en la experiencia, con la cual posibilita efectuar mediciones y conocer el grado de satisfacción.Las opciones de respuestas contienen cuatro niveles de medición: (Muy satisfactorio, satisfactorio, ocasionalmente satisfactorio, y raramente satisfactorio) cada una de estas, representadas por los números 1, 2, 3 y 4 respectivamente.

La población de estudiantes del Curso de Nivelación 2017 (2) es de 2798 y se aplica el reactivo a 1852 estudiantes de la matriz y extensión de Chone del Curso de Nivelación de la ULEAM 2017 II muestra que representan el $66 \%$ del total de estudiantes.

Siguiendo con lo planificado, la comisión encargada de la evaluación de satisfacción de la Gestión de la Coordinación Académica del Curso de Nivelación 2017 (2) previo a la aplicación del reactivo a los estudiantes matriculados y que culminaron mencionado curso, ejecutaron según se detallan, las siguientes actividades:

Diseño de reactivo, presentación y autorización de aplicación de encuestas, Entrega de reactivo vía correo electrónico a cada uno de los encuestados,Llenado y devolución del reactivo a la Coordinación Académica del DANU, Tabulación de la información por parte del equipo de supervisores/ras del DANU,Análisis de datos y elaboración de informes, Presentación del informe al Coordinador Académico del DANU.

\section{Resultados}


A continuación se exponen los resultados de las encuestas, todo ello de manera general, luego de aquello, en cada uno de los aspectos se presentarán las tablas y su descripción respectiva, tabla 1.

\begin{tabular}{|c|c|c|c|c|c|}
\hline \multicolumn{6}{|l|}{ Encuesta de satisfacción docente del curso de nivelación 2017 II } \\
\hline \multicolumn{6}{|l|}{ Organización } \\
\hline Opciones & 1 & 2 & 3 & 4 & Total \\
\hline Organización del curso de nivelación & 114 & 262 & 803 & 673 & 1852 \\
\hline Condiciones del aula para el aprendizaje & 674 & 321 & 328 & 434 & 1757 \\
\hline Cantidad de estudiantes por curso & 116 & 219 & 434 & 294 & 1063 \\
\hline Horario del curso & 245 & 205 & 508 & 680 & 1638 \\
\hline Atención del alumno & 79 & 294 & 873 & 487 & 1733 \\
\hline \multicolumn{6}{|l|}{ Actividad formativa } \\
\hline Los contenidos han cubierto sus espectativas & 30 & 218 & 747 & 771 & 1766 \\
\hline Los temas se han tratado con la profundidad que esperaba & 91 & 329 & 737 & 515 & 1672 \\
\hline Las asignaturas recibidas han completado sus conocimientos & 55 & 184 & 628 & 743 & 1610 \\
\hline La utilidad de los temas aprendidos & 55 & 105 & 732 & 953 & 1845 \\
\hline \multicolumn{6}{|l|}{ Evaluación global } \\
\hline El curso recibido es útil para la información & 42 & 84 & 390 & 1055 & 1571 \\
\hline
\end{tabular}

Tabla 1 Satisfación docente del curso de nivelación 2017.

En la figura 1 se analizó la organización general del Curso de Nivelación, para esto, de los 1852 estudiantes encuestados, 1476 se pronunciaron entre muy satisfactorio y satisfactorio, dicho de otra manera, el $80 \%$ de los encuestados, encontraron más aspectos positivos que negativos en el momento de calificar la organización. Hablar que 8 de cada 10 personas estuvieron conformes con los aspectos que involucran a la organización en general, son cantidades significativas, sin embargo, los dos porcentajes deberían ser el objeto de estudio puesto que para los autores, sería interesante conocer que motiva a calificar de una determinada manera.

En lo referente a las condiciones del aula para el aprendizaje, 995 estudiantes, el 54\% del total de encuestados ubicó la calificación entre ocasionalmente satisfactorio y raramente satisfactorio, inclinándose la balanza hacia una ponderación en los aspectos negativos. Ahora bien, en contraparte se tuvo que 762 estudiantes que representan al $41 \%$ del total de estudiantes, se pronunciaron en el sentido 
de que encontraron más aspectos positivos que negativos. Finalmente 95 estudiantes que representan al $5 \%$ no respondieron, figura 2.

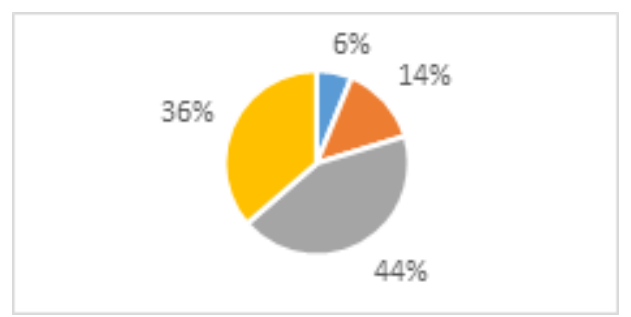

Figura 1. Organización del curso de nivelación



Figura 2. Condiciones del aula para el aprendizaje

En lo relativo al tema de la cantidad de estudiantes por curso (fig. 3), 728 estudiantes, el 39\% del total de encuestados, se revelan más aspectos positivos que negativos, no así en los 335 estudiantes que apenas representan el $18 \%$ del total, encontraron que los aspectos negativos superan a los positivos, ahora, 789 estudiantes no respondieron a este cuestionamiento, debe resaltarse que este grupo representó al $42 \%$ de los encuestados que consideraron no pronunciarse de ninguna manera y que a partir de los resultados expuestos, los investigadores debieron interpretarlos, figura 3.

En lo relativo al horario del curso (fig. 4), 1188 estudiantes que representaron al $64 \%$ del total de encuestados, su respuesta resultó con inclinación hacia lo positivo, de alguna manera todos ellos consideraron que existieron mayores aspectos positivos. De igual manera, 450 estudiantes, el $24 \%$ del total, apuntaron hacia lo negativo, ubicando sus respuestas entre ocasionalmente y raramente satisfactorio, grupo minoritario, no obstante digno de ser investigado; al igual que, el 12\% de estudiantes que no consignaron respuesta alguna para este aspecto. 


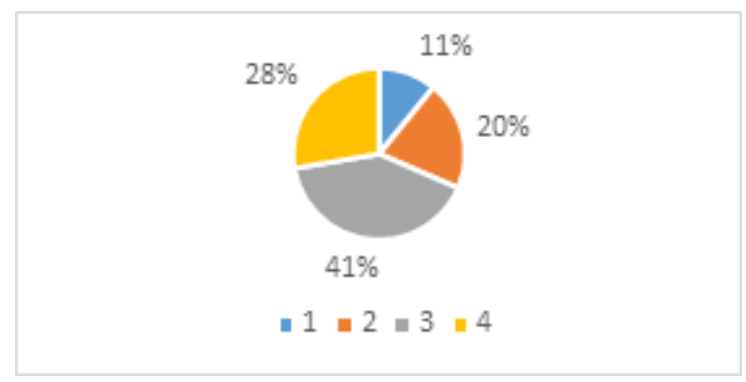

Figura 3. Cantidad de estudiantes por curso

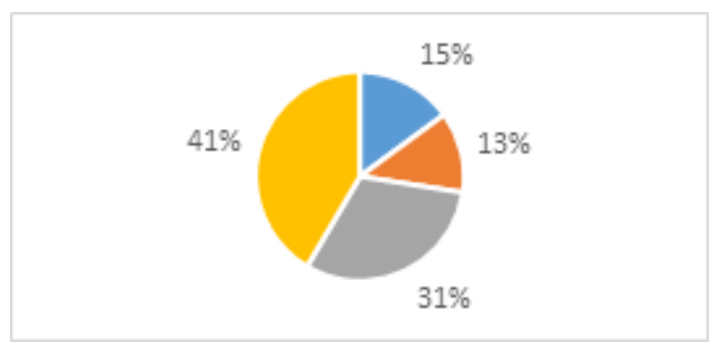

Figura 4. Horario del curso

La percepción de los estudiantes en el tema de la atención que recibieron para solventar sus necesidades, se obtuvieron los siguientes resultados: de los 1852 que son el total de estudiantes encuestados, 1360 que representan el $73 \%$ sientieron que sus necesidades mayoritariamente son satisfechas, no así, los 373 estudiantes o, el $20 \%$ que de acuerdo a sus experiencias, dieron una calificación ponderada hacia lo negativo. Finalmente 119 estudiantes representados por el $6 \%$ que no se pronunciaron ni positivamente ni negativamente, o sea, no respondieron en este aspecto, figura 5.

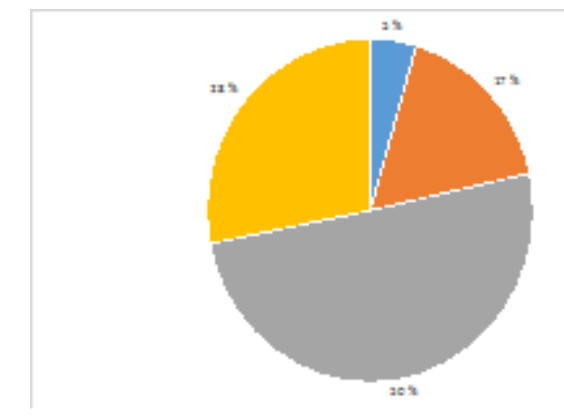

Figura 5 Atención que reciben para solventar sus necesidades.

En torno a la actividad formativa y específicamente en lo que respecta a los contenidos y si aquellos cubrieron las expectativas de los estudiantes (fig. 6); 1518 que representan al 82\% del total de 
encuestados respondieron afirmativamente, sintieron que los contenidos estuvieron alineados a sus necesidades de nivelación; 248 estudiantes que son el 13\% de los encuestados encambio en función de sus experiencias calificaron negativamente a los contenidos, grupo del cual se deberá recabar la información necesaria a fin de determinarse aquellos puntos débiles en el tema contenidos. Se debe destacar además que 86 estudiantes, el 5\% no colocaron respuesta alguna.

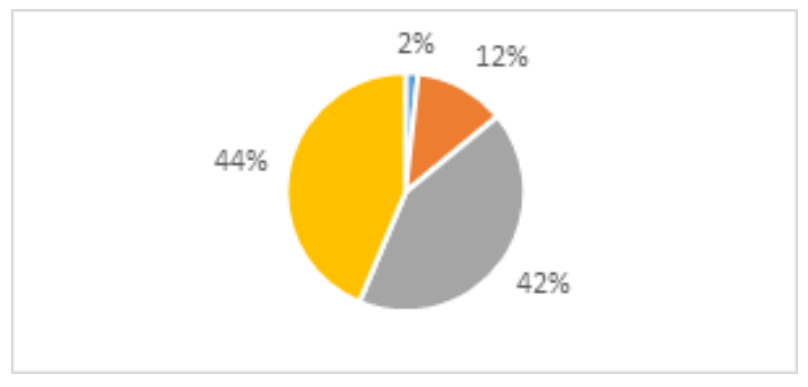

Figura 6. Los contenidos han cubierto sus expectativas.

De la profundidad con que se hayan tratado los temas en el proceso de nivelación (fig. 7), a pesar de que 1252 estudiantes que representan el $67 \%$ del total, calificaron que existen más aspectos positivos que negativos, de aquellos, 515 estuvieron completamente satisfechos y 737 no estuvieron completamente satisfechos, fenómeno digno de estudiarse a fin de establecer aquellos puntos discordantes. Así mismo, 420 estudiantes, representados por el $23 \%$ dieron a conocer que se pudo profundizar en los temas y finalmente, 180 estudiantes, o sea, el 10\% no respondió en esta pregunta.

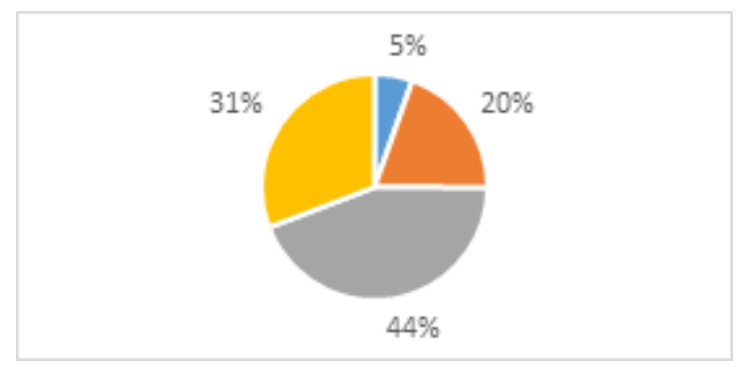

Figura 7. Los temas se han tratado con profundidad esperada.

En cuanto a que si las asignaturas actualizaron sus conocimientos, los estudiantes respondieron de la siguiente manera: 1371 estudiantes, o el 74\% de 1852 estudiantes, inclinaron su respuesta hacia los aspectos positivos, dando a entender su conformidad con la actualización de conocimientos, mientras 
tanto, 239 estudiantes, $13 \%$ del total se encontraron entre medianamente y nada satisfechos, y, 242, el $13 \%$ del total de estudiantes, no respondieron en ninguna de las alternativas, figura 8.

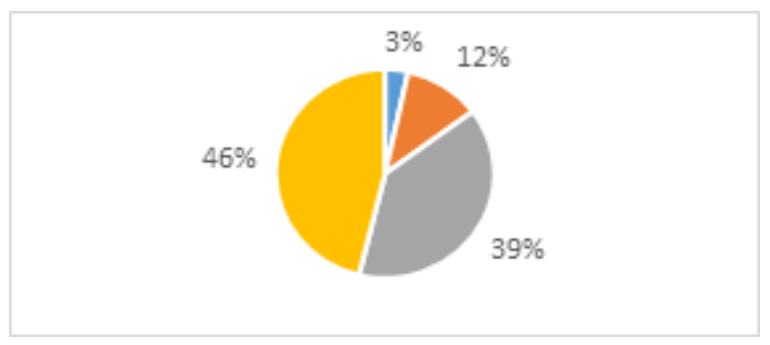

Figura 8. Las asignaturas complenentaron sus conocimiento

En lo relacionado a la utilidad de los temas aprendidos (fig. 9), 1685 estudiantes, el 91\% del total, estuvieron mayormente conformes con la utilidad de los contenidos, no así 160 estudiantes que son el $8,7 \%$. Al respecto de los que no respondieron, apenas 7 estudiantes que representan al $0.3 \%$ del total, se inhibieron de responder ni positiva ni negativamente. En las respuestas del curso hablando de forma general fue de utilidad (fig. 10), 1445 o sea el 78\% respondieron su conformidad; apenas 126 que son el $7 \%$ contestaron que la inconformidad es mayor; de esto, 281 estudiantes, el $15 \%$ no entiendieron la pregunta o demostraron su nulo interés en el tema.

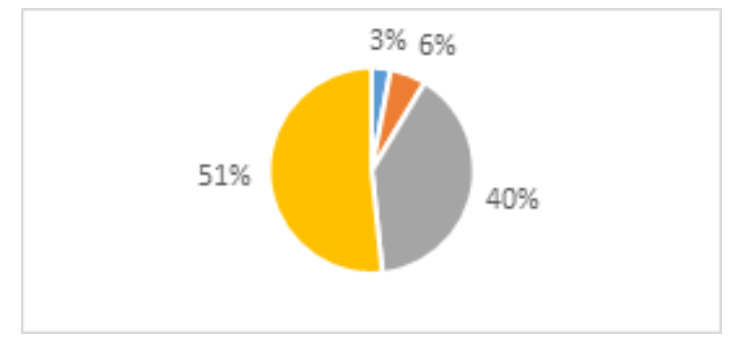

Figura 9. Utilidad de los temas aprendidos

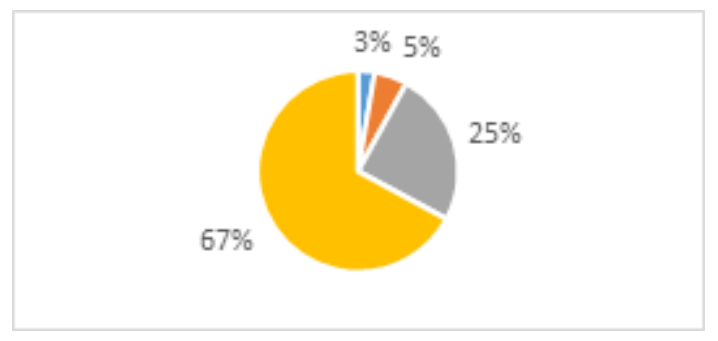

Figura 10. Utilidad del curso 


\section{Discusión}

En lo que se refiere a la organización, se observa que 8 de cada 10 estudiantes encuentran satisfacción en la organización, lo que expresa el éxito del trabajo realizado, no obstante, cabe resaltar que al momento de responder en el apartado de recomendaciones, de 376 estudiantes que corresponden al $20 \%$ del total y que calificaron como ocasionalmente y raramente satisfactorio, tan solo se presenta una recomendación “Se ponga mayor atención en la organización al inicio de la nivelación”, sin que se específiquen los aspectos de inconformidad, se concuerda con otros estudios que hacen alusión al protagonismo y el compromiso del profesorado y la institución en el proceso de implementación del curso (Picado, 2018).

En lo concerniente a las condiciones del aula, se debe mencionar como antecedente que la ULEAM y con ello el DANU sufrieron daños estructurales de importancia en el evento natural ocurrido el 16 de abril de 2016 motivo por el cual en el año de la evaluación, su capacidad estuvo limitada para recibir a 2798 estudiantes, ahora bien, ante esta realidad, se coordinó con las unidades académicas y estas permitieron el uso de sus aulas; pese a que se procuró adecuarlas, estas no tuvieron la capacidad para albergar a un promedio de 40 estudiantes por aula y en muchos casos ni los medios tecnológicos para que su aprendizaje sea óptimo.

Ante tal realidad, del ambiente de aprendizaje, todos aquellos aspectos que interactúan con el estudiante y que resultan favorables para su proceso de aprendizaje. No estuvieron en condiciones adecuadas. Se concuerda con Errázuris (2015), en la que hace alusión a las condiciones materiales, espaciales, visuales, sonoras y olfativas- debería ser una prioridad, no solamente para contribuir a generar una atmósfera más adecuada que favorezca a los procesos de enseñanza y aprendizaje, sino también para enriquecer la educación más allá de sus propósitos académicos.

En este estudio se concuerda que en lo concerniente el espacio educa, enseña, comunica, forma, libera, modela, habla, ríe, llora, invita a vivir, a soñar, a estudiar. El espacio tiene un lenguaje de libertad, de autonomía,de actividad, todo depende de la bondad de cada elemento, del atractivo de las instalaciones, (Heras, 2001). Este autor resalta la importancia de las condiciones físicas de un salón de clases, la incidencia de los objetos sobre los sujetos, entonces, resulta lógico que el producto en los procesos de enseñanza aprendizaje del DANU se pudieron haber visto afectados. 
En lo referente al tema de las condiciones del aula, se pone en contexto la siguiente información recabada de la Secretaría del Vicerrectorado Académico de la "Matriz, de requerimientos para el curso de nivelación 2017 (2)" y es así que el Internet, computadores y equipamento audiovisual en un aproximado del $70 \%$ de aulas no contaron con estos elementos, o sea, de cada 10 paralelos, tan solo 3 de ellos contaban con tecnología. Se concuerda con Ramírez (2010), las TIC han llegado a ser uno de los cimientos básicos de la sociedad, ya que su uso es vital en la educación.

Hablar de las condiciones del aula también es aludir además a las TIC en los contextos educativos.En el estudio el $56 \%$ de encuestados, hacen referencia a más aspectos positivos que negativos y cabe reflexionar entonces, sobre las condiciones en la comunicación y las TIC, en las que se educaron en los niveles anteriores a la nivelación puesto que al parecer, marca en su termómetro signos de conformidad ante esta realidad, por lo que se concuerda con Bartolomé (2001) señala que los recursos informático, audiovisual, tecnológicos, del tratamiento de la información y los que facilitan la comunicación, fortalecen las actividades académicas. Otros autores también hacen alusión a lo planteado anteriormente (Bautista y Alba, 1997).

Según Salavarrieta (2009), la escuela debería integrar nuevos paradigmas culturales: alfabetización digital, fuente de información, instrumento de producción para desarrollar trabajos, material didáctico, instrumento cognitivo, etc., fehacientemente la escuela debería acercar a los estudiantes a la cultura de hoy, no la cultura de ayer. Con estas reflexiones y ante la realidad expuesta en los parrafos anteriores, se puede advertir que la era digital como tal, no ha llegado a la ULEAM, además, está lejos de formar parte del aula y ser una herramienta en el interaprendizaje.

Las condiciones del aula además de estar determinadas por el equipamiento tecnológico, fueron influyentes otros factores como la climatización y cantidad de estudiantes por curso, versus el tamaño del aula. Se conicide con otros estudios que revelan que cualquier deficiencia en el número o tamaño de los espacios disponibles para la enseñanza puede afectar negativamente la conveniencia educativa (Castaldi, 1974). En el caso de los estudiantes de la ULEAM cabe preguntarse, en que medida afectó al rendimiento escolar de los estudiantes y así mismo al rendimiento de los docentes si ninguno de estos factores fueron favorables como se indicó en la información rescatada de la matriz de requerimientos para el curso de nivelación 2017 (2). 
Así mismo en mencionada matriz, se reporta que en este aspecto de la cantidad de estudiantes aceptados para la nivelación en la ULEAM se distribuyeron de la siguiente manera: El 11\% de los paralelos tenían 35 estudiantes; el 54\% contaba con 40 estudiantes; el 18\% de los paralelos, 45 estudiantes en cada uno; finalmente, el $2 \%$ tenían 50 estudiantes, se debe indicar que el 15\% que no se ubica en ninguna de los porcentajes, es porque la matriz de dónde se recaba la información, no fue actualizada en su momento. Se concuerda con Santos (1993), plantea que vivimos y trabajamos en las escuelas sin percatarnos de la influencia que los espacios tienen en los miembros de la comunidad escolar.

Resulta evidente la sobrepoblación para el inicio de clases (2380 estudiantes), problema que en algunos casos disminuyó conforme avanzó la nivelación. Aulas sobrepobladas que pese a la buena actitud del docente, le era difícil atender de forma personalizada a aquellos estudiantes que así lo requerían. Se concuerda con Fraga (2019), en la que afirma que independientemente de las estrategias pedagógicas que se adopten, la cantidad es influyente en la calidad del aprendizaje, además considera que para el desarrollo de destrezas, control de disciplina, entre otros la cantidad óptima estaría entre 20 y 25 estudiantes por aula.

El estudio confirma una sobrepoblación de estudiantes en los diferentes paralelos, existe un $31 \%$ que no ubican una mala la puntuación en esta pregunta. Inclusive allan más aspectos positivos. Por cuanto, el sesgo de respuesta, es en el que los encuestados tienen la tendencia de estar de acuerdo con todas las preguntas ISMARU (2016), desde la óptica de los investigadores, los aspectos como el expresado se imponen en el momento de responder a las preguntas. Una actitud pasiva frente a la crítica, sin que se cuestione el status quo, para muchas personas significa, librarse de problemas futuros.

Otro aspecto es el horario, de los 1852 estudiantes encuestados, 1638 calificaron el horario y la atención recibida. De aquellos que calificaron, 1188 que corresponde al $72 \%$ responden entre muy satisfactorio y satisfactorio; seguramente por cuanto se establecieron tres franjas horarias en las cuales de acuerdo a sus necesidades geográficas fueron ubicados. En contraparte, el 28\% responden entre ocasionalmente y raramente satisfactorio y El $12 \%$ no califica ni efectúan ninguna recomendación, estos últimos porcentajes inevitables por los posibles estudiantes descontentos que sin vivir en la ruralidad, aspiraban un horario diferente al asignado.

En relación con el tratamiento de los contenidos, se concuerda con Diaz Barriga (1992), destaca lo planteado por Bourdieu (1990), relata que conforme a los avanzances en el sistema educativo, existe 
una mayor libertad para ajustar las temáticas por trabajar a las condiciones de los alumnos, sin que se equivoque el autor, esto es justamente lo que suscedió en el proceso de establecimiento de los contenidos para el proceso de nivelación 2017 (2), docentes DANU y docentes representantes de las unidades académicas involucradas, trabajaron conjuntamente para establecer los contenidos.

En lo referente a los contenidos, su pertinencia, profundidad y utilidad. En la pertinencia, del total de evaluados, 1766 respondieron de la siguiente manera: 1518 estudiantes, o sea, el $85.47 \%$ califica entre muy satisfactorio y satisfactorio; mientras que 248 estudiantes que representan el $14.53 \%$ calificaron entre satisfactorio y raramente satisfactorio. Se concuerda con otra investigación en relación a los contenidos curriculares que se enseñen tienen que ser reconocidos como valiosos por los estudiantes, así como por sus familias y la comunidad (Blanco, 2007).

En cuanto a la profundidad de los temas, se coincide con Coll (1987), en la que hace alusión a las capacidades que los alumnos deben ir adquiriendo a lo largo de una etapa o ciclo educativo. El autor asevera que el contenido, su pertinencia y profundidad, fueron el fruto del concenso, cuyos objetivos pretendían simplemente nivelar. Se debe reconocer la confusión en estudiantes al respecto, sus expectativas pudieron estar en función de la carrera profesional elegida. De los 1672 estudiantes que respondieron, el $75 \%$ en otras palabras siete de cada diez estudiantes indicaron que los contenidos fueron tratados con la profundidad esperada; no así el 25\% dos de cada diez estudiantes consideró lo contrario.

De que si las asignaturas complementaron o no sus conocimientos, como en las anteriores respuestas, de 1485 respuestas, ocho de cada 10 estudiantes consideran que si, mientras que uno de cada diez estudiantes opinan estar no tan seguro de aquello; es necesario mencionar a los lectores que por al menos tres ocasiones los contenidos sufrieron modificaciones debido al alto índice de notas bajas que daban cuenta de los problemas que tenían los estudiantes.

\section{Conclusiones}

La investigación realizada genera varias conclusiones, un de ellas es sobre la realidad cultural de quienes optaron por un cupo en la educación superior, un alto porcentaje de estudiantes, se refirieron positivamente a ciertos aspectos evaluados, aspectos en los cuales las autoridades tienen conciencia de que no existen o si existen, no funcionan de una forma adecuada. Cabe reflexionar al respecto, cuáles 
fueron las condiciones en las cuales se desarrolló su educación anterior y en que medida distorcionó su percepción de calidad y de allí generar un sesgo en sus respuestas.

El estudio desarrollado permitió mostrar que no existieron las condiciones didácticas mínimas en lo concerniente a los medios con que cuentan maestros y estudiantes, para así facilitar el proceso de aprendizaje; no se contó con la capacidad física para albergar a la estudiantes aceptados, en consecuencia, la calidad de la educación en la nivelación debió verse afectada al no contar con la infraestructura, los recursos didácticos y tecnológicos mínimos indispensables.

Con la intención de retroalimentar los procesos de evaluación, se debe seguir investigando sobre la idoneidad en la elaboración del reactivo, realizar una autocrítica al respecto de las preguntas, y si estas debieron abarcar aspectos específicos; por ejempo: las condiciones del aula, adecentamiento (condición de paredes, pintura, cantidad, tipo y tamaño de ventanas; cantidad, estado de puertas; condición y tipo de piso; aseo, iluminación de aulas; acceso y aseo de baños, entre otros), sobre otros espacios fuera del aula (pasillos, áreas verdes, etc,) segmentos que no fueron mencionados en la encuesta.

Se confirma que se debe seguir fortaleciendo la selección de maestros, el liderazgo del coordinador académico, la actitud proactiva del equipo de coordinación, la supervisión como parte del proceso de seguimiento, el acompañamiento del vicerrectordo en la solución de problemas inesperados de índole administrativo, el cumplimiento de horarios de forma estricta, entre otros, actuaron como contrapesos a la hora de alcanzar los objetivos. los contrapesos fueron la estrategia elegida en esta administración.

Las autoridades insistieron permanentemente en el abandono de prácticas de la llamada escuela tradicional, no obstante, desde la arquitectura de las aulas, la cantidad de estudiantes, la indisponibilidad de de recursos didácticos y tecnológicos e inclusive el modelo educativo mismo, eran los insumos de aquel modelo educacional.

Deben existir prioridades, entre ellas está el mejorar las condiciones materiales, didácticas, tecnológicas, arquitectónicas, etc., que ineludiblemente enriquecen el objeto mismo de la educación. La administración eficiente, eficaz y efectiva, contribuirá al abandono de prácticas de la llamada escuela tradicional, mediante el establecimiento de renovados paradigmas, que se constituirán en la piedra angular del desarrollo y crecimiento institucional, reflejadosenlos resultados de todos quienes integran la comunidad educativa, siendo de especial atención los estudiantes. 


\section{Bibliografía}

Bautista, A. y Alba, C. (1997) "¿Qué es Tecnología Educativa?: Autores y significados”. Píxel-bit. Recuperado de: https://recyt.fecyt.es/index.php/pixel/article/view/61102/37116

Bartolome, A. (2001). Universidades en la red. ¿universidad presencial o virtual? En Crítica, LII (896), 34-38. Recuperado de: http://www.lmi.ub.edu/personal/bartolome/articuloshtml/2002_AB critica.pdf

Blanco, R., Astorga, A., Guadalupe, C., Hevia, R., Nieto, M., et al. (2007), Educación de calidad para todos: un asunto de derechos humanos. Documento de discusión sobre políticas educativas en el marco de la II Reunión Intergubernamental del Proyecto Regional de Educación para América Latina y el Caribe (EPT/PRELAC). Recuperado de: https://www.oei.es/

Castaldi, B. (1974). Diseño de centros educativos. México: Editorial Pax México.

Coll, C. (1987). Psicología y currículum. Ed. Paidós. Barcelona.

Díaz, A. (1992). "Docentes, planes y programas de estudio e institución educativa. Perfiles

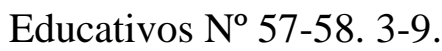

Errázuriz, L. (2015). Calidad Estética del Entorno Escolar: El (f)actor Invisible. Arte, Individuo y Sociedad, 27(1), 81-100. Recuperado de: https://bit.ly/2KYZ8IW

Fraga J. (2019). El número de alumnos en las aulas influye en el aprendizaje. La Hora. Pag. A2.

García, I. (2010). Sistema de evaluación. Recuperado de: https://www.eumed.net/libros-gratis/2010b /687/index.htm

Heras, L.(2001). Espacios culturales y educativos”. Servicio de Publicaciones e Intercambio científico de la Universidad de Málaga.

ISMARU (2016). Sesgos de respuesta en la encuesta (web log post). Recuperado de: https://blog.encuesta.com/sesgos-de-respuesta-en-la-encuesta/

Moreno, T. (2016). Evaluación del aprendizaje y para el aprendizaje. Mexico. UAM 
Palladino, E y Palladino, L (1998). Administración Organizacional. Argentina. Espacio.

Picado, M. (2018). Sugerencias didácticas para la implementación de un curso de nivelación en matemáticas: La sistematización de una experiencia en la Universidad Nacional de Costa Rica.Revista Educare, 22,(3). Recuperado de: https://www.scielo.sa.cr/pdf/ree/v22n3/1409-4258$\underline{\text { ree-22-03-314.pdf }}$

Ramírez, R. C. (2010), “Las TICS en el Aula”, NNTT, Innovación y experiencias educativas. No $45-6^{\mathrm{a}}, \mathrm{p} 2$.

Salavarrieta, L. (2009). Importancia de las Tics en la educación, Herramientas didácticas en ambientes virtuales de aprendizaje. SED-UNAD. Bogotá.

Santos, G. (1993.). Espacios escolares. Recuperado de: https://bit.ly/2E24CRT 